\title{
Simulation of low angle X-ray diffraction on multilayers subjected to diffusion
}

\author{
A.G. Fedorov \\ Institute for Single Crystals, Lenin Ave. 60, 61001 Kharkov, Ukraine \\ fedorov@xray.isc.kharkov.comd
}

\begin{abstract}
Calculative method based on the Riccatti type differential equation was tested for simulation of low angle X-ray diffraction patterns from the one-dimensionally ordered multilayer. Some peculiarities of diffraction were revealed connected with asymmetrical distortion of the multilayer profile due to different processes on the layer boundaries.
\end{abstract}

Keywords: multilayers, X-ray diffraction

Paper received 12.09.00; revised manuscript received 13.10.00; accepted for publication 12.12.00.

\section{Introduction}

Thin film multilayers find application in different areas, for example, as dispersive elements in X-ray optics, or objects with inherent quantum dimensional features in a case of single-crystalline multilayers (superlattices). Xray diffraction examination of the structure of multilayers reveal the appearance of additional peaks (satellites) near the Bragg reflections for the single-crystalline multilayers and low angle diffraction reflections near the incident beam for both amorphous (or fine grain) and single-crystalline multilayers. First type of diffraction is connected with periodic deviations of the unit cell parameter and electron density, and second type of diffraction is caused only by periodic deviations of electron density of alternating layers.

Multilayer efficiency in different applications depends among others on the interface abruptness and flatness as well as on stability of the structure. The diffusion intermixing and formation of new phases on the boundaries may occur at elevated temperature [1,2]. Low angle $\mathrm{X}$ ray diffraction on multilayers with comparatively large period is described commonly using the set of recurrent relations based on the Fresnel equation solved on interfaces $[3,4]$. This supposes the uniform dielectric constant by layer and abrupt interface transitions. Real multilayer may not offer such ideal rectangular profile. Usually, the let-out is to approximate the smoothed profile as a set of lamella. In this work another approach based on solution of Maxwell equations for layered structure with arbitrary profile of the dielectric constant distribution is examined.

\section{Background}

Assume the plane wave passing through the medium as outlined in Fig. 1, where $X Y$ plane separates vacuum and medium, $H$ and $k$ lies in XZ plane and $E$ is parallel to $Y$ axis.The simplified Maxwell equations for this case are

$$
\begin{array}{ll}
\frac{\partial H_{x}}{\partial z}-\frac{\partial H_{z}}{\partial x}=-i \frac{\omega}{c} \varepsilon E_{y} & \frac{\partial H_{x}}{\partial x}+\frac{\partial H_{z}}{\partial z}=0 \\
\frac{\partial E_{y}}{\partial z}=-i \frac{\omega}{c} H_{x}, \frac{\partial E_{y}}{\partial x}=i \frac{\omega}{c} H_{z} & \frac{\partial E_{y}}{\partial y}=0
\end{array}
$$

The plane wave expression $E=E_{0} \exp i(k r)=E_{0} \times$ $\times \exp i\left(k_{x} r_{x}+k_{y} r_{y}+k_{z} r_{z}\right)$ put in (1) yields the following values for $H_{x}, H_{z}$ :

$H_{x}=-\frac{c}{\omega} k_{z} E_{y}, \quad H_{z}=\frac{c}{\omega} k_{x} E_{y}$

Now, conformably to [4], let us turn out the following. Equations for incident wave propagating in medium at the angle $\varphi$ may be written as

$$
\begin{aligned}
& E_{y}^{P}=P(z) \exp i q, H_{x}^{P}=-n P(z) \cos \varphi \exp i q, \\
& H_{z}^{P}=n P(z) \sin \varphi \exp i q
\end{aligned}
$$

and equations for a reflected wave are

$$
\begin{aligned}
& E_{y}^{R}=R(z) \exp i q, H_{x}^{R}=n R(z) \cos \varphi \exp i q, \\
& H_{z}^{R}=n R(z) \sin \varphi \exp i q
\end{aligned}
$$




\section{A.G. Fedorov: Simulation of low angle X-ray diffraction on multilayers ...}

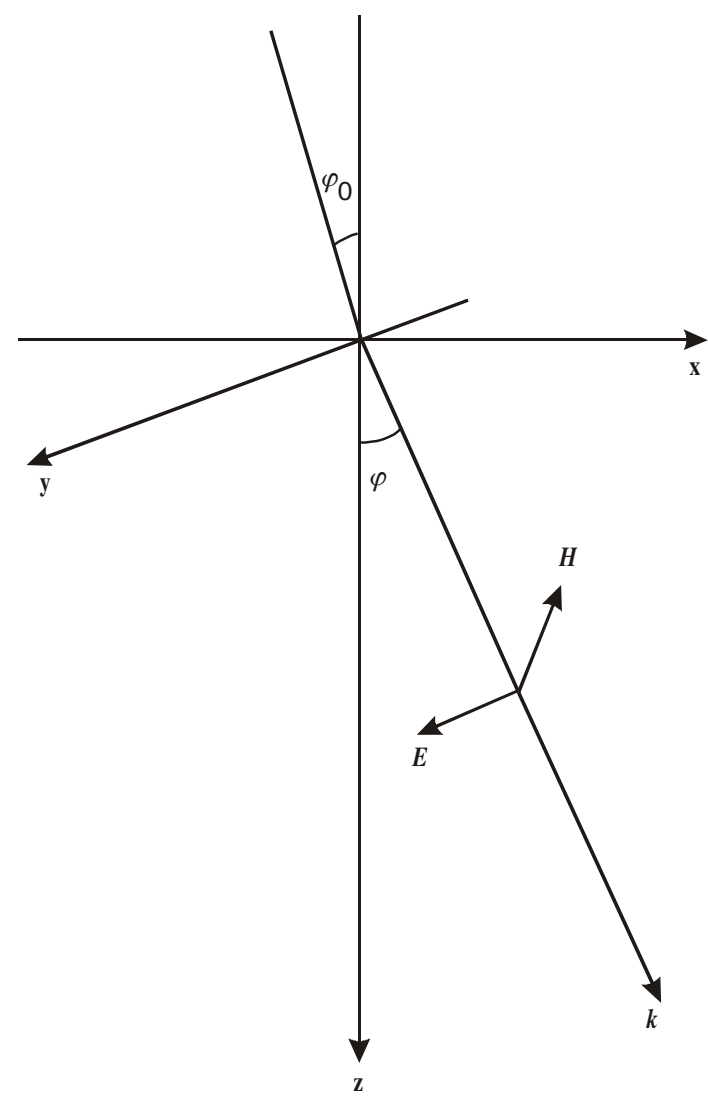

Fig. 1. X-ray plane wave.

Here we take in account that $c / \omega=1 / k_{0}$ in vacuum, $k=k_{0} n=k_{0} \sqrt{\varepsilon(z)}, \varepsilon(z)$ is the dielectric constant of medium, and hence $H_{x}^{P}=-\frac{c}{\omega} k_{z} E_{y}^{P}=-\frac{k \cos \varphi}{k_{0}} P(z) \exp i q$ and so on. $P(z)$ and $R(z)$ are the amplitudes of initial and reflected waves, correspondingly, $q$ denotes $k x \sin \varphi$. Then, applying (1) to (2) and (3), we obtain the system

$$
\begin{aligned}
& \frac{d R}{d z}-\frac{d P}{d z}-(P-R) \frac{\varepsilon^{\prime}}{2 \varepsilon \cos ^{2} \varphi}+i k \cos \varphi(P-R)=0 \\
& \frac{d R}{d z}+\frac{d P}{d z}-i k \cos \varphi(P-R)=0
\end{aligned}
$$

Here $\varepsilon^{\prime}=d \varepsilon / d z$. Denoting reflectivity $X=R / P$, we turn out from (4) after some transforms

$$
\frac{d X}{d z}=-2 i k X \cos \varphi+\left(1-X^{2}\right) \frac{\varepsilon^{\prime}}{4 \varepsilon \cos ^{2} \varphi}
$$

This Riccati type equation describes reflection from the medium with arbitrary distribution of dielectric constant and gives the diffraction pattern in appointed angular range. For X-ray wave range the following more convenient form is applicable. Meaning that the refraction coefficient $n(z)=\sin \varphi_{o} / \sin \varphi$, we get

$$
\begin{aligned}
& \frac{d X}{d z}=-2 i k_{o} X \sqrt{\varepsilon(z)-\sin ^{2} \varphi_{o}}+ \\
& +\left(1-X^{2}\right) \frac{\varepsilon^{\prime}(z)}{4\left[\varepsilon(z)-\sin ^{2} \varphi_{o}\right]}
\end{aligned}
$$

In the $\mathrm{X}$-ray waverange, $\varepsilon(z)$ is defined as $\varepsilon=1-\delta+i \gamma$, where

$\delta=\frac{e^{2}}{m c^{2}} \frac{\lambda^{2}}{\pi} N_{a} f_{1}, \gamma=\frac{e^{2}}{m c^{2}} \frac{\lambda^{2}}{\pi} N_{a} f_{2}$,

$\lambda$ is the wavelength, $N_{a}$ is the atomic density in the unit cell, $f_{1}$ and $f_{2}$ are real and imaginary parts of atomic scattering factor, respectively, that may be taken from [5] or downloaded from www.cxro.lbl.gov/optical_constants/ asf.html.

Considering $\varepsilon=$ const in (6), one can get obviously the usual solution for the film with uniform dielectric constant on the substrate $d X / d z=-2 i k_{0} X \sqrt{\varepsilon-\sin ^{2} \varphi_{0}}$ and $X=X_{0} \exp \left(-2 i k_{0} h \sqrt{\varepsilon-\sin ^{2} \varphi_{0}}\right)$ where $h$ is the film thickness and initial condition $X_{0}$ is the known Fresnel eqution for reflection from the boundary of two media

$X_{0}=\frac{\sqrt{\varepsilon_{f}-\sin ^{2} \varphi_{0}}-\sqrt{\varepsilon_{s}-\sin ^{2} \varphi_{0}}}{\sqrt{\varepsilon_{f}-\sin ^{2} \varphi_{0}}+\sqrt{\varepsilon_{s}-\sin ^{2} \varphi_{0}}}$

These are just the set of equations that allow to calculate the reflection from the rectangular profile multilayer mentioned above.

Change of multilayer profile due to diffusion may be described by applying the solution of diffusion equation

$$
\varepsilon(z, t)=\varepsilon_{0} \sum_{n} a_{n} \exp \left(\frac{-n^{2} \pi^{2} D t}{H^{2}}\right) \cos \left(\frac{n \pi z}{H}\right)
$$

where $\varepsilon_{0}$ is the amplitude value of dielectric constant deviation, namely, the difference between constants of one and another layers; $H$ is the period of multilayer, $D-$ diffusion coefficient, $t$-duration of the process. In the most simple case we suppose the rectangular initial profile

$\varepsilon(z, 0)=\varepsilon_{0} \sum_{n} a_{n} \cos \left(\frac{n \pi z}{H}\right)$

with expansion coefficients

$a_{0}=S, a_{n}=\frac{2}{n \pi} \sin (n \pi S)$

that undergoing to identical diffusion smoothing on the both boundaries of layers, which is supposed on Fourier 
expansion by cosines. $S$ denotes a symmetry factor of the period, or the relation of the layer thickness to the period. If the layer thicknesses are equal, then $S=0.5$. Thus, the profile smoothing due to diffusion is defined by the dependence of expansion coefficients on $\mathrm{Dt}$ :

$$
a_{n}(t)=a_{n}(0) \exp \left(-n^{2} \pi^{2} D t / H^{2}\right)
$$

\section{Results}

Described approach was tested by calculating the diffraction on the hypothetical Mo-Si multilayer consisting of 30 periods with period value $10 \mathrm{~nm}$. Dielectric constant profile was represented as the Fourier series according to (8) and equation (6) solved numerically by the 4th order Run-ge-Kutta method with initial conditions given by (7) on the boundary of silicon substrate and first layer. Solution procedure was executed from the boundary to the multi-layer surface where reflectivity was taken as a product of complex conjugated amplitudes $X$. This was repeated in a sequence of angular points. The resulting curve was convoluted with instrumental broadening function of Gaus-sian shape and the diffraction pattern so was obtained. In Fig. 2 there are presented the calculated diffraction patterns of rectangular profile multilayer depending on variation of its symmetry factor. Here and below shown are the reflections from the 2 nd to 6 th order.

Periodically arranged layers with alternating average electron densities represent the one-dimension lattice for which the structure amplitude may be calculated as a Fourier transform of electron density distribution. Squares of structure amplitudes obtained in such manner are presented in Table. These values are proportional to the intensities of corresponding reflections and may be compared with diffraction patterns in Fig. 2. In this particular case of rectangular multilayer profile, calculation with conventional method using the Fresnel equations gives completely the same patterns. More attractive task is to simulate diffraction pattern for multilayer subjected to diffusion intermixing and thus the profile smoothing. Moreover, the processes on the layer bounda-

Table. Squares of structure amplitudes for different symmetry factors

\begin{tabular}{c|ccc}
\hline \hline \multirow{2}{*}{$\begin{array}{c}\text { Reflection } \\
\text { order }\end{array}$} & \multicolumn{3}{|c}{ Symmetry factor } \\
\cline { 2 - 4 } & 0.34 & 0.45 & 0.5 \\
\hline 1 & 100 & 100 & 100 \\
\hline 2 & 23.21 & 2.45 & 0 \\
\hline 3 & 0.06 & 9.04 & 11.11 \\
\hline 4 & 6.66 & 2.21 & 0 \\
\hline 5 & 3.41 & 2.05 & 4 \\
\hline 6 & 0.06 & 1.86 & 0 \\
\hline \hline
\end{tabular}

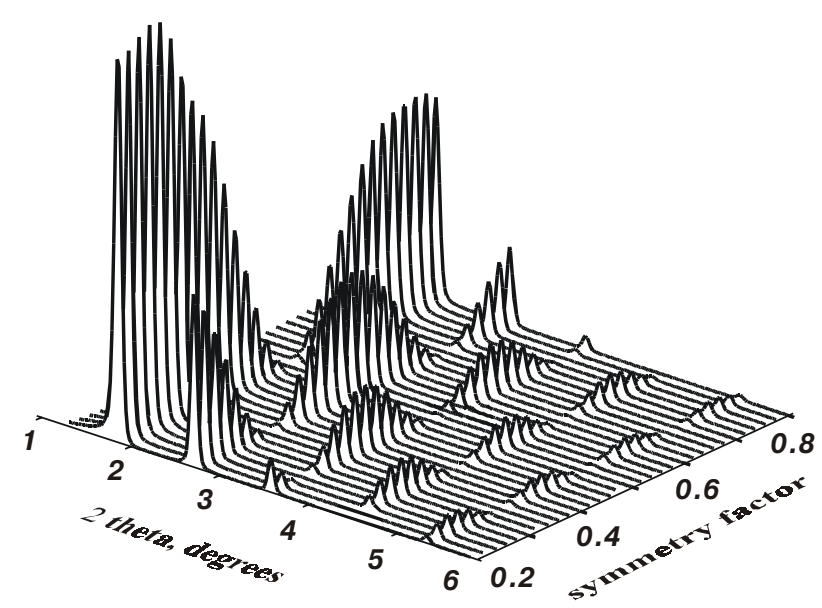

Fig. 2. Evolution of diffraction pattern depending on the symmetry factor.

ries may not be obligely identical, so the asymmetrical profile transformation may take place $[1,6]$. A set of calculated diffraction patterns for multilayer in a course of symmetrical diffusion smoothing is shown in Fig. 3. Teen the comprehensible exponential decrease of reflection intensities is obvious, it is more fast for reflections with a larger number. But diffusion with different coefficients on the boundaries gives another development of diffraction picture. This is demonstrated in Fig. 4. A slight decrease of the layer symmetry leads to appearance and growth of formerly forbidden reflections.

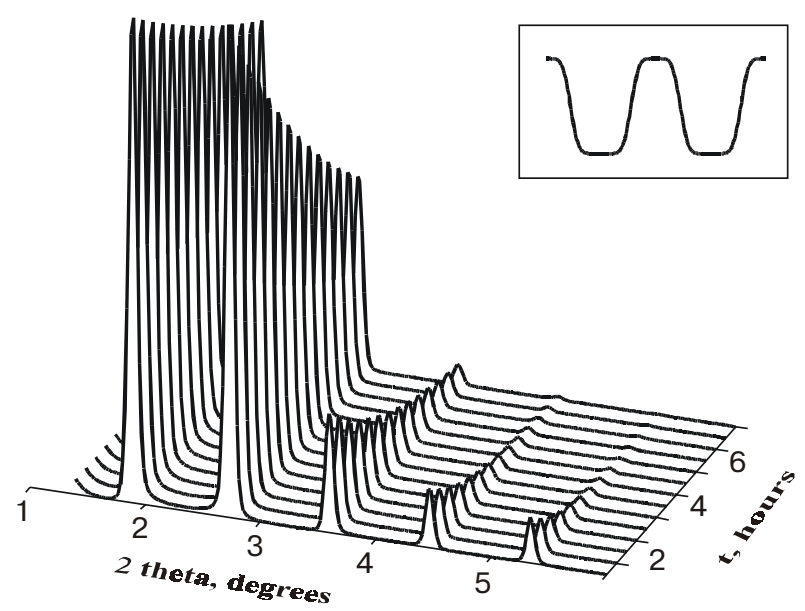

Fig. 3. The set of calculated diffraction patterns of the multilayer subjected to symmetrical diffusion intermixing with diffusion coefficient $2.5 \cdot 10^{-19} \mathrm{~cm}^{2} / \mathrm{s}$, symmetry factor 0.45 . Final profile is shown on the inset.

SQO, 3(4), 2000 
A.G. Fedorov: Simulation of low angle X-ray diffraction on multilayers ...

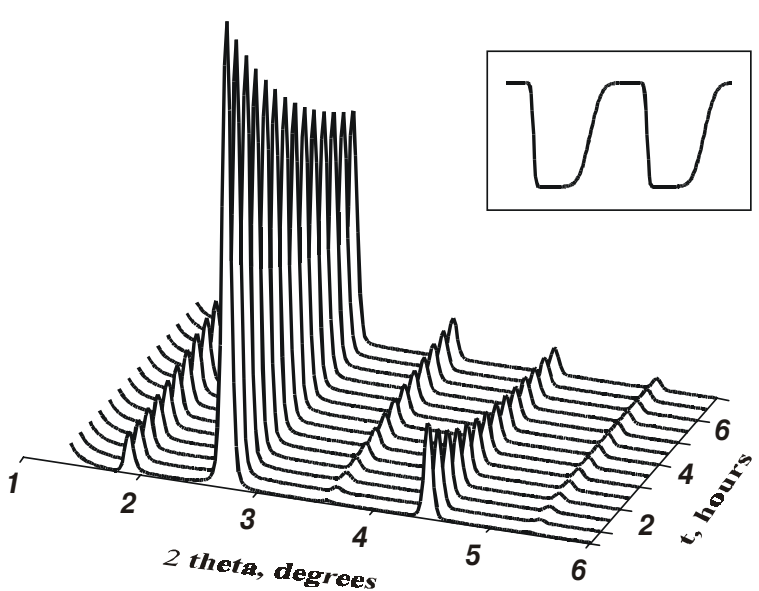

Fig. 4. The set of calculated diffraction parrerns of the multilayer after simulated asymmetric diffusion intermixing with diffusion coefficients $2 \cdot 10^{-20} \mathrm{~cm}^{2} / \mathrm{s}$ and $5 \cdot 10^{-19} \mathrm{~cm}^{2} / \mathrm{s}$ on the sides of layer. Symmetry factor is 0.5 .

\section{Conclusions}

Some peculiarities of low angle diffraction patterns from multilayers are caused by its profile asymmetrical distortion during processes of intermixing or phase forma- tion at the boundaries. Assumed mechanisms of multilayer profile transformations may be verified by simulation of diffraction pattern solving numerically the equation (6). In general, any distribution of dielectric constants of the layer or periodical multilayer may be introduced in a suitable form and then the diffraction pattern of this object may be simulated. If, especially, the investigation of asymmetrical diffusion is of interest, then the preliminary calculation of diffraction pattern is useful because it allows to find the relation of layer thicknesses the most sensitive to expected effect.

\section{References}

1. K. Holloway, K. B. Do, and R. Sinclair. J. Appl. Phys. 65(2), pp. 474-480 (1989).

2. J. M. Freitag and B. M. Clemens. Appl. Phys. Lett. 73, pp. 43-45 (1998).

3. A.V. Vinogradov and I.V. Kozhevnikov, Multilayer X-ray mirrors, in X-ray optics, edited by A. N. Orajevsky // Proc. P. N. Lebedev Inst. 196, pp.62-102. Moscow, Publishing House «Nauka», 1989 (in Russian).

4. D.L. Windt. Computers in Physics 12, pp.360-370 (1998).

5. L.M. Brechovsky. Waves in Stratified Media, Moscow, Publishing House of the Academy of Science, USSR 1957 (in Russian).

6. R.S. Rosen, D.G. Stearns, M.E. Kassner, J.-I. Koike, Y. Cheng, and S.P. Vernon, Nanostruct. Mater. 3, pp. 195-199 (1993). 